\title{
Relationships Exploration for Real-time Static Voltage Stability Prediction of Power Systems
}

\author{
Songkai Liu \\ School of Electrical Engineering \\ Wuhan University \\ Wuhan, China \\ e-mail:1612234945@qq.com
}

Cheng Tian

School of Electrical Engineering

Wuhan University

Wuhan, China

e-mail:397000712@qq.com

Huimin Qiu

Beijing Electric Power Corporation

Beijing, China

\author{
Xinyuan Cui \\ School of Electrical Engineering \\ Wuhan University \\ Wuhan, China \\ Dong Chen \\ School of Electrical Engineering \\ Wuhan University \\ Wuhan, China \\ $\mathrm{Li} \mathrm{Tu}$ \\ School of Electrical Engineering \\ Wuhan University \\ Wuhan, China
}

\begin{abstract}
An approach based on data mining and relationships exploration is presented for estimating the static voltage stability relative margin of power systems. The data set is created based on simulations by the software PSS/E. The input variables selected for estimation are corresponding to the relationships highly ranked by MIC and PPMCC. These relationships are also shown and some of them are explained from the perspective of power system operation. If the measured values of these variables are obtained from wide area measurement system (WAMS), the relative margin can be estimated in real time since its relationships with these variables are explored. The approach is tested on a 39-bus system provided by PSS/E and illustrative results indicate the scheme is accurate and effective.
\end{abstract}

Keywords-Maximal information coefficient (MIC);Pearson product-moment correlation coefficient (PPMCC); relationships exploration; static voltage stability.

\section{INTRODUCTION}

Static voltage stability is a major concern for secure operation of power systems. It is important to find out the voltage collapse point and to know the distance from the current operating point to it [1]. Various techniques are reported to identify and estimate the maximum load ability in the research of static voltage stability [2]-[3]. One of the conventional techniques is solving the power flow repetitively, starting at a base load leading up to the collapse limit [4], [5], in which the load and the generator output are increased in steps. However, this paper doesn't adopt these specific methods, but simulates the increase of load and generator output using Python language in the software PSS/E. In this way, not only a batch of power flow at different operating points can be solved at one time, but also different increase directions of load and generator output can be freely selected.
The estimation of static voltage stability relative margin for a system is based on data mining methods in [6]-[9]. Data mining is a technique that evaluates knowledge or rules through the database, and it is much more difficult to interpret complicated data as the size of database becomes larger. In the research of relative margin estimation, a statistical tool [10] for data mining is adopted in this paper, which is different to the conventional methods used in [6]-[9]. The tool can detect novel associations in large data sets, and it is based on the maximal information coefficient (MIC) [10] and the Pearson product-moment correlation coefficient (PPMCC) [11].

This paper proposes a voltage stability estimation scheme, which is shown in Fig. 1. The scheme will be more desirable than artificial neural networks, expert systems and some other current techniques when applied to different kinds of systems since the input features are selected based on data mining and relationship exploration, not the fixed ones proposed by some papers.

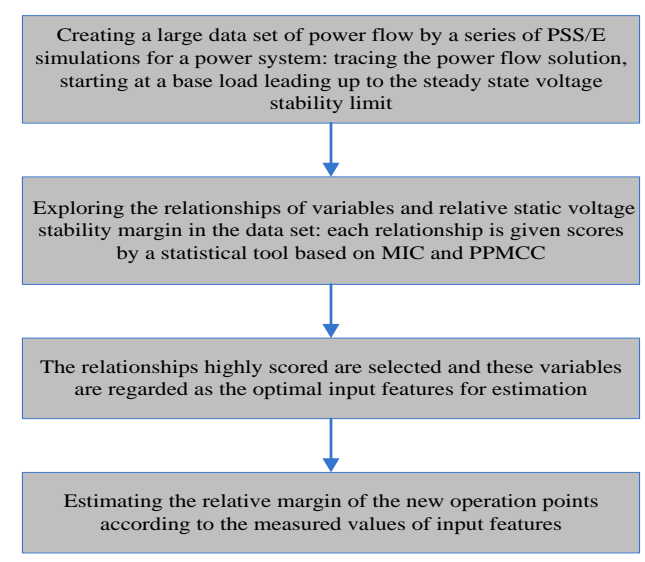

Figure 1. Estimation scheme. 


\section{PRoblem ST ATEMENT AND SUPPORT ING MATHEMATICAL METHODS}

\section{A. Static Voltage Stability Relative Margin}

Load active power margin can be directly obtained from the curve $P-V$ [12] as illustrated in Fig. 2. The initial active power consumed by the load is $P_{0}$ and the maximum value of active power can be transferred is $P_{\max }$. The load active power margin and static voltage stability relative margin $M$ are defined as (1) and (2) respectively.

$$
\begin{aligned}
& \Delta P=P_{\max }-P_{0} \\
& M=\frac{\Delta P}{P_{\max }} * 100 \%
\end{aligned}
$$

The collapse limit and the active power margin depend on how the load and generator output increase. In Fig. 3, different initial operating points, increase directions of load and generator output, collapse limit points, and the system static voltage stability boundary are shown in a twodimensional space.

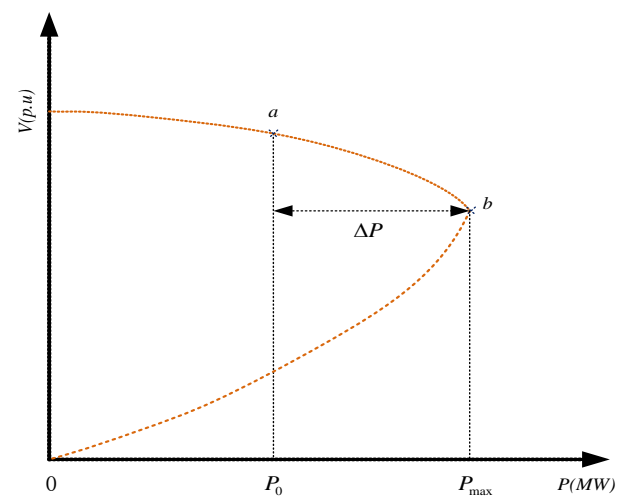

Figure 2. Traditional PV diagram.

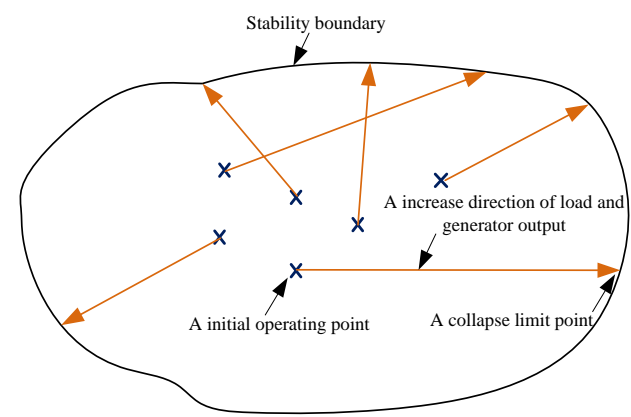

Figure 3. Different collapse limit points due to increase directions of load and generator output for possible initial operating points.

\section{B. $M I C$}

Given a finite set $D$ of ordered pairs, the $x$-values of $D$ are partitioned into $x$ bins and the $y$-values of $D$ are partitioned into y bins, allowing empty bins. Such a pair of partitions can be called an $x_{\text {-by- }} y$ grid. Giving a grid $G$, let $\left.D\right|_{G}$ be the distribution induced by the points in $D$ on the cells of $G$. The distribution on the cells of $G$ is obtained by letting the probability mass in each cell be the fraction of points in $D$ falling in that cell. For a fixed $D$, different grids $G$ result in different distributions $\left.D\right|_{G}$ [10]. For a data set $D$ of two-variable, the MIC of their relationship is given by (3), (4). The interested reader can refer to [10] a more detailed description.

For a finite set $D \subset R^{2}$ and positive integers $x, y$,

$$
I^{*}(D, x, y)=\max I\left(\left.D\right|_{G}\right)
$$

Where the maximum is over all grids $G$ with $x$ columns and $y$ rows, and $I\left(\left.D\right|_{G}\right)$ denotes the mutual information of $\left.D\right|_{G}$. The MIC of two-variable data with sample size $\mathrm{n}$ and grid size less than $B(n)$ is given by

$$
\operatorname{MIC}(D)=\max _{x y<B(x, y)}\left\{\frac{I^{*}(D, x, y)}{\log \min \{x, y\}}\right\}
$$

$$
\text { Where } \omega(1)<B(n) \leq O\left(n^{1-\varepsilon}\right) \text { for some } 0<\varepsilon<1 \text {. }
$$

In the research, $B(n)=n^{0.6}$ is used because it's found to work well after test [10]. MIC falls between 0 and 1 .

\section{CReating A Large Dat a Set of Power Flow BASED ON SIMULATION}

This paper uses Python programming language in PSS/E to achieve the follows: increasing load and generator output by steps in a setting direction; solving the power flow of every operation point until the Jacobian matrix is singular and power flow is not convergent. The flow chart is illustrated in Fig. 4.

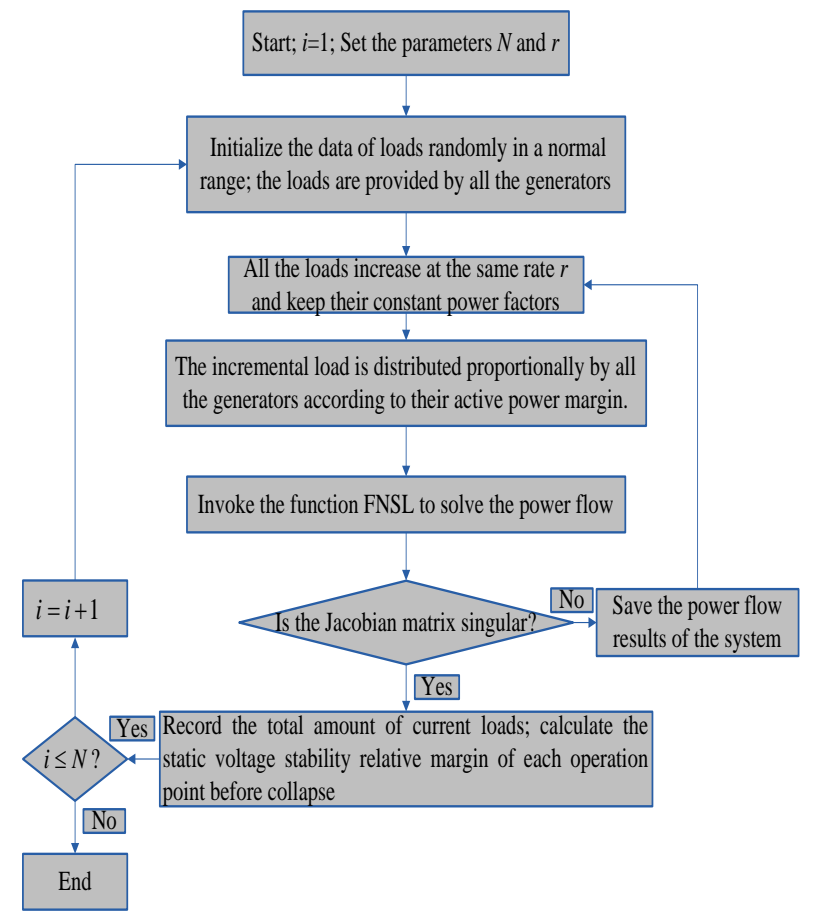

Figure 4. Flow chart for creating a large data set of power flow based on simulation in PSS/E.

In the paper, a 39-bus test system [13] provided by PSS/E is used, which is shown in Fig. 5. 


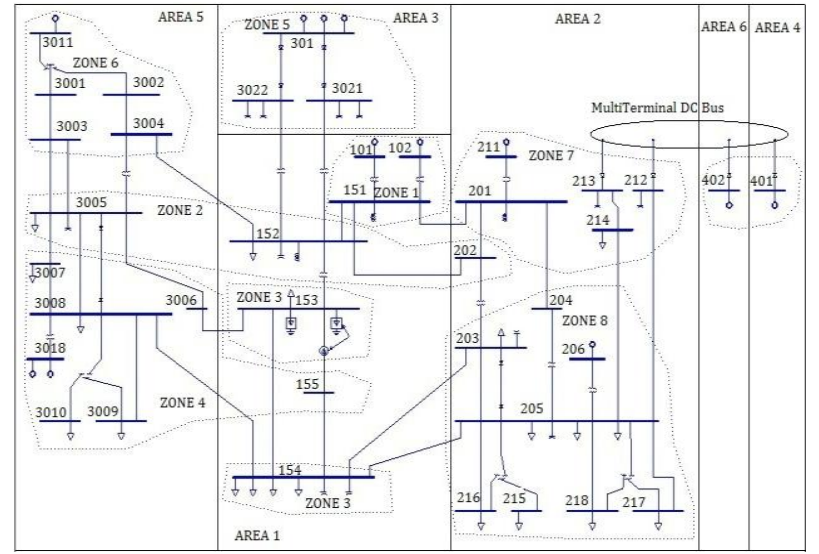

Figure 5. 39-bus test system provided by PSS/E.

\section{EXPLORINGTHE RELATIONSHIPS OF VARIABLES AND} RELATIVE ST ATIC VOLT AGE ST ABILITY MARGIN

\section{A. Top Relationships Explored by MIC and PPMCC}

The relationships between the relative margin and variables are given scores and ranked by MIC and PPMCC. Table I shows the top $2 \%$ of relationships by MIC and Table II shows the top $2 \%$ of relationships by PPMCC. The rank given by MIC is different to the one given by PPMCC. The relationships highly ranked by MIC don't necess ary to have high degrees of linearity.

TABLE I. TOP $2 \%$ OF RELATIONSHIPS BY MIC

\begin{tabular}{|c|c|c|c|c|c|}
\hline Var1 & Var2 & MIC & $\begin{array}{l}\text { MIC } \\
\text { Rank }\end{array}$ & PPMCC & $\begin{array}{l}\text { PPMCC } \\
\text { Rank }\end{array}$ \\
\hline Margin & Q3001_3003 & 0.919 & 1 & -0.944 & 113 \\
\hline Margin & Q3001_3WNDTR & 0.919 & 2 & 0.944 & 114 \\
\hline Margin & $Q 151 \_101$ & 0.911 & 3 & 0.900 & 188 \\
\hline Margin & $Q 212 \_205$ & 0.908 & 4 & -0.842 & 219 \\
\hline Margin & $I \% 206 \_205$ & 0.908 & 5 & -0.840 & 223 \\
\hline Margin & $V 151$ & 0.907 & 6 & 0.885 & 190 \\
\hline Margin & S151_102 & 0.907 & 7 & -0.839 & 227 \\
\hline Margin & $P G 101$ & 0.906 & 8 & -0.838 & 232 \\
\hline Margin & PG102 & 0.906 & 9 & -0.838 & 230 \\
\hline Margin & $P G 206$ & 0.906 & 10 & -0.838 & 233 \\
\hline
\end{tabular}

TABLE II. TOP $2 \%$ OF RELATIONSHIPS BY PPMCC

\begin{tabular}{llcccl} 
Var1 & Var2 & PPMCC & $\begin{array}{l}\text { PPMCC } \\
\text { Rank }\end{array}$ & MIC & $\begin{array}{l}\text { MIC } \\
\text { Rank }\end{array}$ \\
\hline Margin & Q202_152 & -0.962 & 1 & 0.894 & 74 \\
\hline Margin & O203 & 0.966 & 2 & 0.847 & 176 \\
Margin & O3007 & 0.960 & 3 & 0.847 & 177 \\
\hline Margin & Q153_UQFC & -0.960 & 4 & 0.831 & 221 \\
Margin & Q203_202 & -0.960 & 5 & 0.877 & 105 \\
\hline Margin & P3005_3003_1 & 0.960 & 6 & 0.871 & 120 \\
Margin & P3005_3003_2 & 0.960 & 7 & 0.871 & 121 \\
\hline Margin & P3003_3001 & 0.959 & 8 & 0.874 & 112 \\
Margin & P3003_3005_1 & -0.959 & 9 & 0.865 & 130 \\
Margin & P3003_3005_2 & -0.959 & 10 & 0.865 & 131
\end{tabular}

From the perspective of power system, reasonable explanation can also get for the relationships detected. For example, the relationship between the variable Q3001_3003 and the relative marg in is the top 1 in Table I. Q3001_3003 represents the reactive power from bus 3001 to bus 3003 and the relationship is shown in Fig. 6. With the increase of all the loads, the static voltage stability relative margin of the system will decrease. The reactive power demand at bus 3005 increases and it leads to the increase of reactive power output of the generator at nearby bus 3011 . Therefore the reactive power from bus 3001 to bus 3003 will increase, which can be understood with the help of Fig. 5.

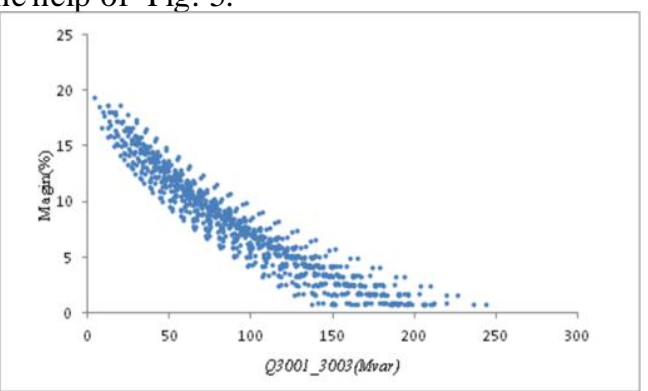

Figure 6. Scatter plot of the relative margin and the variable Q3001_3003.

\section{B. MIC versus PPMCC}

Fig. 7A shows that MIC versus PPMCC for all pairwise relationships in the data set. In fact, $\mathrm{MIC}$ also tends to give a high MIC score to lots of relationships with high $|\rho|$. In different areas of Fig. 7A, different kinds of relationships can be found. Some examples are as follows.

1) Fig. 7B: Both PPMCC and MIC yield low scores for unassociated variables. It indicates no specific relationship exist between the variable I\% 153_152_152 and the relative margin.

2) Fig. 7C: Ordinary linear relationships score high under both MIC and PPMCC tests. It indicates an obvious linear relationship between the variable Q202_152 and the relative margin.

3) Fig. 7D: Relationships detected by MIC but not by PPMCC, because the relationships are nonlinear. It indicates a kind of nonlinear relationship between the variable the variable QG206 and the relative margin.

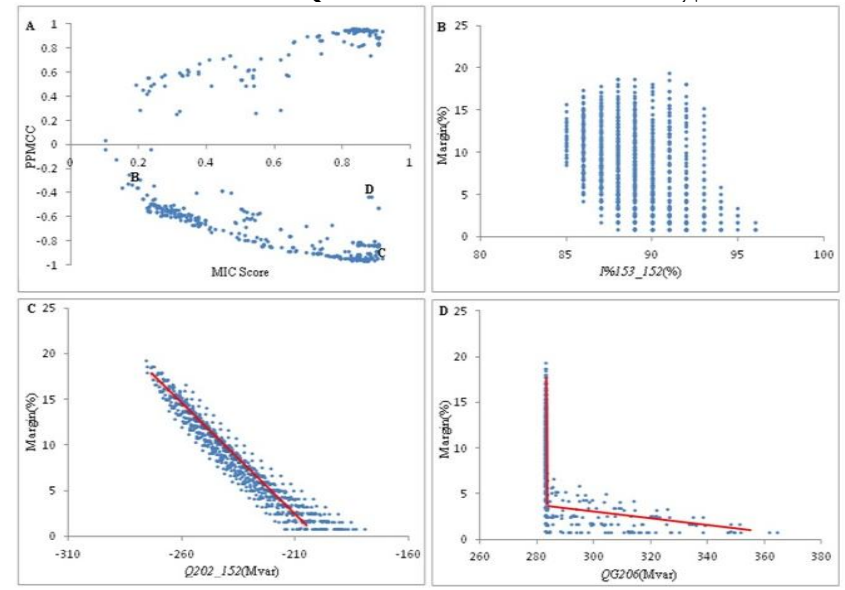

Figure 7. Application of MIC and PPMCC to the data set. (A) MIC versus PPMCC for all pair-wise relationships in the data set. (B)(D) Examples of relationships from (A). 


\section{Top Nonlinear Relationships}

In order to discover the nonlinear relationships of variables and the relative marg in comprehensively and quickly, the statistic MIC- $\rho^{2}$ is adopted. For a relationship, a larger MIC and a smaller $|\rho|$ get a larger MIC- $\rho^{2}$. The statistic MIC- $\rho^{2}$ is small for linear relationships and large for nonlinear relationships. Table III shows the top $1 \%$ of relationships by MIC- $\rho^{2}$, and all the relationships are shown in Fig. 8. The variable QG3018 is always the same with the variable Q3018_3008, so Fig. 8C is also for QG3018.

TABLE III. TOP $1 \%$ OF NONLINEAR RELATIONSHIPS BY MIC- $\rho^{2}$

\begin{tabular}{|c|c|c|c|c|c|}
\hline Var 1 & Var2 & MIC- $\rho^{2}$ & & MIC & PPMCC \\
\hline Margin & $Q G 206$ & 0.693 & 1 & 0.885 & -0.438 \\
\hline Margin & Q206_205 & 0.685 & 2 & 0.878 & -0.438 \\
\hline Margin & Q3018_3008 & 0.630 & 3 & 0.906 & -0.525 \\
\hline Margin & $Q G 3018$ & 0.630 & 4 & 0.906 & -0.525 \\
\hline Margin & Q3008_3018 & 0.534 & 5 & 0.619 & 0.291 \\
\hline
\end{tabular}
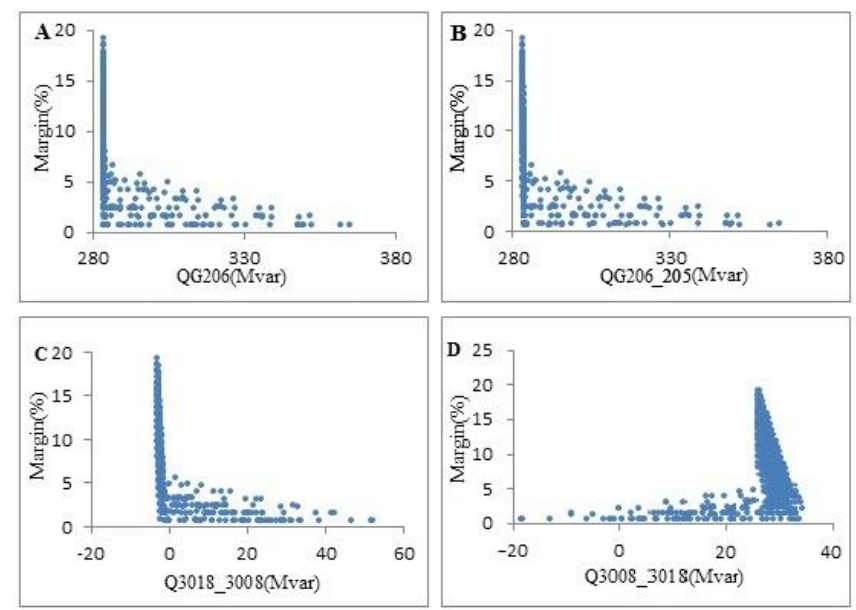

Figure 8. Scatter plot of the top $1 \%$ of nonlinear relationships selected by MIC- $\rho^{2}$.

\section{ESTIMATION OF ST ATIC VOLT AGE ST ABILITY RELATIVE MARGIN AND TEST RESULTS}

\section{A. Program Flow Chart for Estimation of Relative Margin}

For each variable, the estimation method is as follows. As it is illustrated in Fig. 9, the measurement value of the variable Q202_152 is $x_{0}$ when the system is operated at a new point. In all the points in Fig. 9, the smallest value of $x_{\text {-axis is }} X_{\text {min }}$ and the largest value is $X_{\max }$. All the points with $x_{\text {-axis }}$ in the range $\left(x_{1}, x_{2}\right)$ are searched out. The

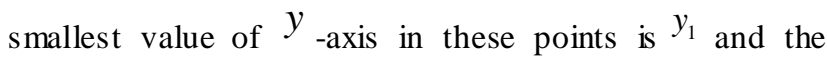
largest value is $y_{2}$. The relative margin of the new operation point can be considered in the range $\left(y_{1}, y_{2}\right)$. Equations (6)-(10) are given in Fig. 9. Specially, 3\% is used in (7) and 0.5 is used in (8), which are found to work well after test. A too wide search range leads to an imprecise estimation result. If the search range is too narrow, maybe no result is returned because the sample size is finite.

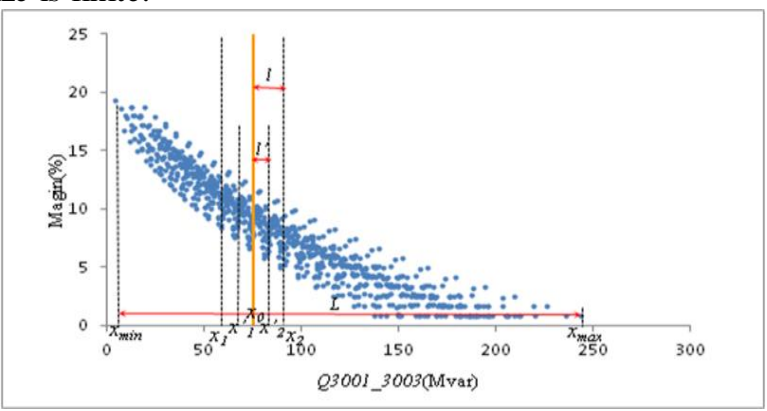

Figure 9. Estimation method for each variable.

$$
\begin{gathered}
L=X_{\max }-X_{\min } \\
l=L^{*} 3 \% \\
l^{\prime}=0.5^{*} l \\
X_{1}=X_{0}-l \\
X_{2}=X_{0}+l
\end{gathered}
$$

The selected variables are used to estimate the relative margin and the program flow chart is shown in Fig. 10.

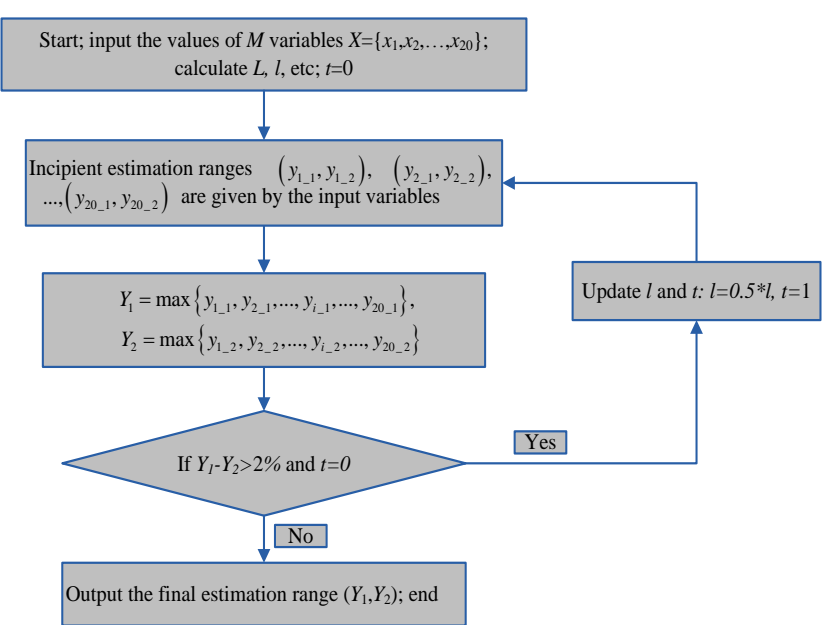

Figure 10. Program flow chart of estimating relative margin.

\section{B. Estimation Results}

The above method is applied to estimate the ranges of relative margin for new operation states. In the tests, 10 possible new operation points are set stochastically. The results are shown in Table IV. Some explanatory annotations for Table IV are as follows.

1) $R$ is the final estimation range of a new point, which is given by the program with the 15 selected variables as input features.

2) $P$ is the span of $R$, which can measure the precision of estimation range to a certain extent.

3) $R_{1}$ is given by the program with 5 variables as input features, which are corresponding to the top $1 \%$ of relationships by MIC. 
4) $R_{2}$ is given by the program with 5 variables as input features, which are corresponding to the top $1 \%$ of relationships by PPMCC.

5) $R_{3}$ is given by the program with 5 variables as input features, which are corresponding to the top $1 \%$ of nonlinear relationships by MIC- $\rho^{2}$.

6) $S$ is the simulation value given by the new tests of load and generator output increase, which is used to verify the correctness of estimation ranges.

TABLE IV. ESTIMATION RANGES OF NEW OPERATION POINTS

\begin{tabular}{lllllll} 
No. $R(\%)$ & $P(\%)$ & $R_{1}(\%)$ & $R_{2}(\%)$ & $R_{3}(\%)$ & $S(\%)$ \\
\hline 1 & $0.85-1.72$ & 0.87 & $0.85-3.31$ & $0.81-3.31$ & $0.85-1.72$ & 1.71 \\
\hline 2 & $5.74-6.56$ & 0.82 & $2.75-7.32$ & $5.74-6.56$ & $2.63-9.76$ & 5.98 \\
\hline 3 & $9.32-10.92$ & 1.60 & $8.70-10.92$ & $9.32-10.92$ & $3.67-15.32$ & 10.26 \\
\hline 4 & $11.02-12.17$ & 1.16 & $10.00-12.61$ & $11.02-12.17$ & $5.5-16.94$ & 11.97 \\
\hline 5 & $1.72-2.50$ & 0.78 & $0.84-4.07$ & $0.88-3.39$ & $1.72-2.50$ & 2.48 \\
\hline 6 & $4.17-6.67$ & 2.50 & $4.17-7.50$ & $3.48-6.67$ & $2.50-8.87$ & 6.61 \\
7 & $9.02-10.83$ & 1.82 & $7.50-11.48$ & $9.02-10.83$ & $0.92-12.90$ & 10.74 \\
\hline 8 & $10.66-12.50$ & 1.84 & $9.17-13.11$ & $10.66-12.50$ & $2.56-14.52$ & 12.40 \\
\hline 9 & $2.50-4.20$ & 1.70 & $1.72-4.20$ & $2.50-5.98$ & $1.72-4.27$ & 3.36 \\
\hline 10 & $6.67-9.24$ & 2.58 & $6.03-9.24$ & $6.67-10.26$ & $1.74-11.29$ & 7.56 \\
\multicolumn{7}{c}{$R_{1}, R_{2}$ and $R_{3}$ can be regarded as 3 control groups for }
\end{tabular}

$R$. The results of $R$ have remarkable precision compared with other groups, which demonstrates appropriate combination of MIC and PPMCC is better than separating them individually. In general, each simulation value $S$ for new operation point is in the range $R$ and this demonstrates the correctness of estimation ranges. $S$ is slightly out of the range in a very few tests, which is due to the finiteness of previous samples. The possibility of $S$ being out of the range $R$ is lower than $1 \%$ after the statistics of more tests, and it obviously decrease with the increase of previous operation samples' total number.

\section{CONCLUSIONS}

The scheme is more intelligent and efficient than some current techniques since the input features are selected fro m a great nu mber of variables based on data mining and relationship exploration, not some fixed ones determined by different attempts of input data combinations. The scheme will be more desirable than current techniques when applied to different kinds of systems since the input features are selected based on data statistics and mining, and its applicability will not be influenced by the change of the structure and scale. The estimation ranges are with high precision, which relies on the total number of previous operation samples to a certain extent. Using this scheme, the system engineers can know the approximate distance from the current operating point to voltage collapse point with the estimation value of relative margin. The relative margin estimation scheme can be an important reference for the security, operation and controlof power system.

\section{REFERENCES}

[1] T. Van Cutsem and C. Vournas, Voltage stability of electric power systems. norwell, MA: Kluwer, 1998.

[2] B. Leonardi, and V. Ajjarapu. An approach for real time voltage stability margin control via reactive power reserve sensitivities, IEEE Trans. Power Syst., vol.28, no. 2, pp. 615-625, May 2013.

[3] B. Leonardi, and V. Ajjarapu. Development of multilinear regression models for online voltage stability margin estimation, IEEE Trans. Power Syst., vol.26, no. 1, pp. 374-383, Feb. 2011.

[4] A. Semlyen, B. Giao, and W. Janischevskj, Calculation of the extreme loading condition of a power system for the assessment of voltage stability, IEEE Trans. Power Syst., vol.6, no. 1, pp. 307315, Feb. 1991.

[5] O. O. Obadina and G. J. Berg, Determination of voltage stability limit in multimachine power systems, IEEET rans. Power Syst., vol 3, no. 4, pp. 1545-1554, Nov. 1988.

[6] T. M. L. Assis, A. R. Nunes, and D. M. Falcao, Mid and long-term voltage stability assessment using neural networks and quasisteadystate simulation, in Proc. Power Engineering, 2007 Large Engineering Systems Conf., Oct. 10-12, 2007,pp. 213-217.

[7] S. Kamalasadan, A. K. Srivastava, and D. Thukaram, Novel algorithm for online voltage stability assessment based on feed forward neural network, in Proc. IEEE Power Eng. Soc. General Meeting, Jun. 18-22, 2006.

[8] H. Innah and T. Hiyama, Neural network method based on PMU data for voltage stability assessment and visualization, in Proc. TENCON 2011-2011 IEEE Region 10 Conf., Nov. 21-24, 2011, pp. 822-827.

[9] I. Herbert and H. Takashi, A real time PMU data and neural network approach to analyze voltage stability, in Proc. APAP, 2011 International Conf., Oct. 16-20, 201 1, pp. 1263-1267.

[10] N. R. David, A. R. Yakir, K. F. Hilary, R. G. Sharon, M. Gilean, J. T. Peter, S. L. Eric, M. Michael, and C. S. Pardis. Detecting novel associations in large data sets, Science, vol.334, pp. 1518-1524, Dec. 2011.

[11] J. Benesty, J. Chen, and Y. Huang, On the importance of the Pearson correlation coefficient in noise reduction, IEEE Trans. Audio, Speech, Lang. Process., vol. 16, no. 4, pp. 757-765, May 2008.

[12] D. Q. Zhou, U. D. Annakkage, and A. D. Rajapakse, Online monitoring of voltage stability margin using an artificial neural network, IEEE Trans. Power Syst., vol.25, no. 3, pp. 1566-1574, Aug. 2010.

[13] Power Technologies, Inc., PSS/E Application Program Interface, Aug. 2004 